\title{
Switchable lasing in multimode microcavities
}

\section{Zhukovsky, Sergei V.; Chigrin, Dmitry N.; Lavrinenko, Andrei; Kroha, Johann}

\section{Published in:}

Physical Review Letters

Link to article, DOI:

10.1103/PhysRevLett.99.073902

Publication date:

2007

Document Version

Publisher's PDF, also known as Version of record

Link back to DTU Orbit

\section{Citation (APA):}

Zhukovsky, S. V., Chigrin, D. N., Lavrinenko, A., \& Kroha, J. (2007). Switchable lasing in multimode microcavities. Physical Review Letters, 99(7), 073902. https://doi.org/10.1103/PhysRevLett.99.073902

\section{General rights}

Copyright and moral rights for the publications made accessible in the public portal are retained by the authors and/or other copyright owners and it is a condition of accessing publications that users recognise and abide by the legal requirements associated with these rights.

- Users may download and print one copy of any publication from the public portal for the purpose of private study or research.

- You may not further distribute the material or use it for any profit-making activity or commercial gain

- You may freely distribute the URL identifying the publication in the public portal

If you believe that this document breaches copyright please contact us providing details, and we will remove access to the work immediately and investigate your claim 


\title{
Switchable Lasing in Multimode Microcavities
}

\author{
Sergei V. Zhukovsky, ${ }^{1, *}$ Dmitry N. Chigrin, ${ }^{1}$ Andrei V. Lavrinenko, ${ }^{2}$ and Johann Kroha ${ }^{1}$ \\ ${ }^{1}$ Physikalisches Institut, Universität Bonn, Nussallee 12, D-53115 Bonn, Germany \\ ${ }^{2}$ COM-DTU and Nano-DTU, Technical University of Denmark, Building 345V, \\ DK-2800 Kongens Lyngby, Denmark
}

(Received 25 January 2007; published 15 August 2007)

\begin{abstract}
We propose the new concept of a switchable multimode microlaser. As a generic, realistic model of a multimode microresonator a system of two coupled defects in a two-dimensional photonic crystal is considered. We demonstrate theoretically that lasing of the cavity into one selected resonator mode can be caused by injecting an appropriate optical pulse at the onset of laser action (injection seeding). Temporal mode-to-mode switching by reseeding the cavity after a short cooldown period is demonstrated by direct numerical solution. A qualitative analytical explanation of the mode switching in terms of the laser bistability is presented.
\end{abstract}

DOI: 10.1103/PhysRevLett.99.073902

PACS numbers: 42.60.Fc, 42.55.Tv, 42.82.Gw

Microlasers with cavity sizes comparable to the radiation wavelength $\lambda$ are very promising from both fundamental and application points of view [1] for use as integrated coherent light sources. Making microlasers capable of multiple-wavelength emission contributes even more towards miniaturization of optical components, and also provides an additional degree of freedom in light control. The common approach towards microlaser tunability is in essence modification of the optical properties of a single-mode cavity by thermal [2], micromechanical [3] or electro-optical means [4].

In this Letter we propose the new concept of a switchable microlaser, composed of a multimode laser microresonator, where lasing can be switched on demand to any of its eigenmodes. While the broad gain profile of semiconductor and dye lasers provides little discrimination between neighboring modes and, thus, leads to mode hopping and multistable mode dynamics, we show that a definite resonator mode can be selected for lasing by injection seeding [5,6], i.e., by injecting an appropriate pulse before and during the onset of lasing, such that the stimulated emission builds up in a chosen mode from this seeding field rather than from the random noise present in the system due to quantum fluctuations and spontaneous emission. We investigate the time needed for switching between different lasing modes and analyze how this process is influenced by noise in competition with the seeding signal.

We generalize the semiclassical multimode laser model of Ref. [7] to study the dynamics of individual modes in a two-mode laser, where an external field is present in the cavity due to the injection seeding. Semiclassical theories of optical amplifiers [5] have been successfully employed to study the mode dynamics in multimode lasers $[7,8]$, especially in the context of laser instabilities [9]. An example of a two-mode microcavity, which we have chosen to study numerically, is depicted in Fig. 1(a). The microcavity is based on two coupled defects in a two- dimensional (2D) photonic crystal (PhC) made of dielectric nanopillars [10,11].

The system of two identical coupled single-mode cavities supports two modes, namely, the bonding (symmetric) and the antibonding (antisymmetric) mode, characterized by spatial field distributions $u_{1,2}(\mathbf{r})$ and frequencies $\omega_{1,2}=$ $\omega_{0} \mp \Delta \omega$, respectively. Here $\Delta \omega$ is the mode detuning from the frequency of the single-cavity resonance, $\omega_{0}$. For weak mode overlap the spatial intensity profiles of

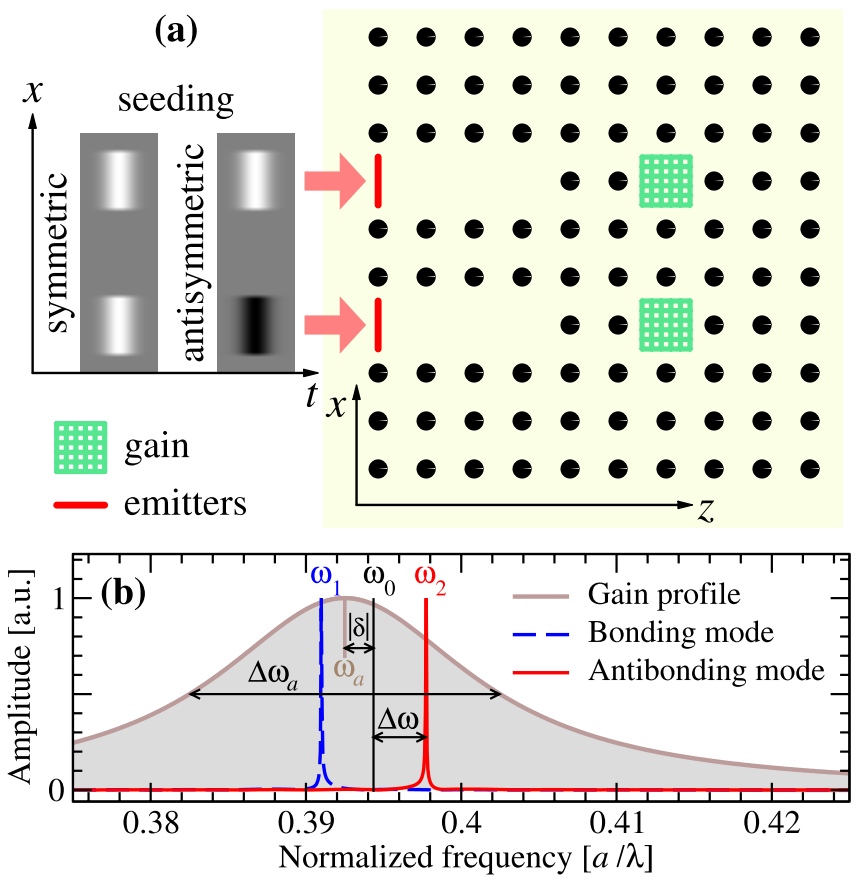

FIG. 1 (color online). (a) Schematic illustration of the resonant system under study. Two coupled defects in a periodic 2D PhC lattice contain a gain medium. Two emitters shown are used to produce the seeding signal delivered to the defect sites by two waveguides. The spatial field distribution of the seeding signal is schematically depicted as a gray scale on the left; (b) frequency parameters of the two-mode laser; see text. 
the two modes nearly coincide, $\left|u_{1}(\mathbf{r})\right|^{2} \approx\left|u_{2}(\mathbf{r})\right|^{2}$. We assume that the cavities contain a laser medium with the gain centered at frequency $\omega_{a}=\omega_{0}+\delta$, homogeneously broadened to have a line width of $\Delta \omega_{a}>\Delta \omega$, where $\delta$ is the detuning of the gain profile from the cavity frequency $\omega_{0}$. See Fig. 1(b) for the definition of the frequency parameters. For the numerical calculations below we take a quadratic array of $12 \times 12$ nanopillars with dielectric constant $\epsilon=9$ in air $(\epsilon=1)$. The lattice period is $a=$ $500 \mathrm{~nm}$ and the pillar radius $r=0.2 a$. Then both defect modes have frequencies near $a / \lambda \simeq 0.395$ [Fig. 1(b)].

To obtain an initial understanding of the seedinginduced mode switching, the semiclassical MaxwellBloch equations [5] may be simplified using the rotatingwave and the slowly varying envelope approximations. All the spatial dependencies of the electric field and atomic polarization can then be represented in the basis of the two cavity modes, such that $E(\mathbf{r}, t)=$ $E_{1}(t) u_{1}(\mathbf{r}) e^{-i \omega_{1} t}+E_{2}(t) u_{2}(\mathbf{r}) e^{-i \omega_{2} t}$, etc., and the atomic polarization can be eliminated adiabatically $[7,8]$. For class-A lasers, where the radiative decay rate $\gamma_{\perp}=$ $\Delta \omega_{a} / 2$, the nonradiative decay rate $\gamma_{\|}$, and the cavity mode decay rates $\kappa_{j}$ are related as $\gamma_{\perp} \gg \gamma_{\|} \gg \kappa_{j}$, the following system of equations for the slowly varying envelopes $E_{j}(t)$ is obtained [7] $(i, j=1,2, i \neq j)$,

$$
\begin{aligned}
\frac{d E_{j}(t)}{d t}= & g R_{j}\left[\left(\mathcal{L}_{j}-\frac{\kappa_{j}}{g R_{j}}\right)-\eta \mathcal{L}_{j}\left(\alpha_{j j}^{j j} \mathcal{L}_{j}\left|E_{j}\right|^{2}\right.\right. \\
& \left.\left.+\left[\alpha_{i i}^{j j} \mathcal{L}_{i}-\alpha_{i j}^{j i} \operatorname{Re}\left(\chi_{j} \mathcal{M}_{j i}\right)\right]\left|E_{i}\right|^{2}\right)\right] E_{j}(t)+F_{j}(t) .
\end{aligned}
$$

In Eqs. (1) the terms linear in $E_{j}(t)$ describe stimulated emission driving and are controlled by the light-matter coupling $g \simeq \sqrt{2 \pi \omega_{0} d^{2} / \hbar}$, with $d$ the dipole moment of the atomic transition, by the pumping rates projected onto the modes $j=1,2, R_{j}=\int_{G} u_{j}^{*}(\mathbf{r}) u_{j}(\mathbf{r}) R(\mathbf{r}) d \mathbf{r}$, and by the cavity mode decay rates $\kappa_{j}$. The coefficients $\mathcal{L}_{j}=\operatorname{Im} \beta_{j}^{-1}$, with $\beta_{1,2}=\delta \pm \Delta \omega-i \Delta \omega_{a} / 2$, account for the different mode-to-gain couplings due to asymmetrical detuning of the atomic transition with respect to the resonator lines. The terms cubic in $E_{j}(t)$ describe field saturation above the lasing threshold, where $\eta=d^{2} / 2 \gamma_{\|} \hbar^{2}$ and the overlap integrals $\alpha_{k l}^{i j}=\int_{G} u_{i}^{*}(\mathbf{r}) u_{j}(\mathbf{r}) u_{k}^{*}(\mathbf{r}) u_{l}(\mathbf{r}) d \mathbf{r}$ are taken over the regions $G$ containing the gain medium. Since $\left|u_{1}(\mathbf{r})\right|^{2} \approx\left|u_{2}(\mathbf{r})\right|^{2}$ we can assume $\alpha_{j j}^{i i}=\alpha_{j i}^{i j}=\alpha, R_{1}=$ $R_{2}=R$, and $\kappa_{1}=\kappa_{2}=\kappa$. The cross-saturation terms, with $\mathcal{M}_{i j}=\beta_{i}^{-1}+\left(\beta_{j}^{*}\right)^{-1}$ and $\chi_{1,2}=-i \gamma_{\|} /( \pm \Delta \omega-$ $\left.i \gamma_{\|}\right)$, depend in an asymmetrical way on the mode indices $i, j$. This asymmetry remains small unless $\Delta \omega \ll \Delta \omega_{a}$.

The inhomogeneous terms $F_{j}(t)$ originate from the external injection seeding field and from a noise field accounting for spontaneous emission [7]. For vanishing functions $F_{j}$, Eqs. (1) would take the form of the standard two-mode competition equations [5] with mode coupling constant $C$ slightly exceeding unity. This corresponds to bistable lasing [12] and to mode hopping in the presence of stochastic noise in the system [13]. If both an external seeding field $\mathcal{E}^{s}(\mathbf{r}, t)$ and a stochastic noise field $\mathcal{E}^{n}(\mathbf{r}, t)$ are present in the cavity, $\mathcal{E}(\mathbf{r}, t)=\mathcal{E}^{s}(\mathbf{r}, t)+\mathcal{E}^{n}(\mathbf{r}, t)$, one obtains

$$
\begin{aligned}
F_{j}(t) & \approx \frac{\omega_{j} \mathcal{L}_{j}}{\tau} \int_{t-\tau}^{t} d t^{\prime} e^{i \omega_{j} t^{\prime}} \int_{G} u_{j}(\mathbf{r}) \mathcal{E}\left(\mathbf{r}, t^{\prime}\right) d \mathbf{r} \\
& =F_{j}^{s} F(t)+F_{j}^{n}(t) .
\end{aligned}
$$

The time integration in Eq. (2) is the averaging over a time interval $\tau$ larger than $1 / \Delta \omega$. The function $F(t)$ is determined by the temporal dependence of $\mathcal{E}^{s}(\mathbf{r}, t)$. The coefficients $F_{j}^{s}$ and $F_{j}^{n}(t)$ are determined by the spatial overlap of each mode with the seeding and noise fields, respectively. We consider the situation when the seeding prevails over the noise, i.e., $F_{j}^{s} F(t) \gg F_{j}^{n}(t)$, before and during the onset of lasing. After the onset the $E_{j}$ become so large that the terms $F_{j}$ have no effect anymore. In this situation the evolution of the resonator will be determined by the ratio of $F_{1}^{s}$ and $F_{2}^{s}$.

We analyze the influence of the balance between $F_{1}^{s}$ and $F_{2}^{s}$ on the final lasing state by numerical solution of Eqs. (1) and plotting the phase trajectories of the temporal resonator state evolution in the $\left(\left|E_{1}\right|^{2},\left|E_{2}\right|^{2}\right)$ plane (Fig. 2). The numerical values of the coefficients in Eqs. (1) are calculated for the model PhC structure of Fig. 1(a). As seen in Fig. 2, the lasing state first reaches overall intensity saturation $\left(\left|E_{1}\right|^{2}+\left|E_{2}\right|^{2}=E_{s}^{2}\right)$ and then drifts towards one of the stable fixed points corresponding to singlemode lasing (either $\left|E_{1}\right|^{2}=E_{s}^{2}$ or $\left|E_{2}\right|^{2}=E_{s}^{2}$ ). The drift occurs after the sharp bend seen in each of the phase trajectories. If the mode coupling constant is $C=1$, the drift becomes infinitely slow, and the $(1,0)-(0,1)$ line in Fig. 2 turns into a line of fixed points. For our case, where $C$ only slightly exceeds unity, the drift happens on a longer time scale than the initial overall intensity growth, and the intermode beats decay fast after the lasing onset. In the
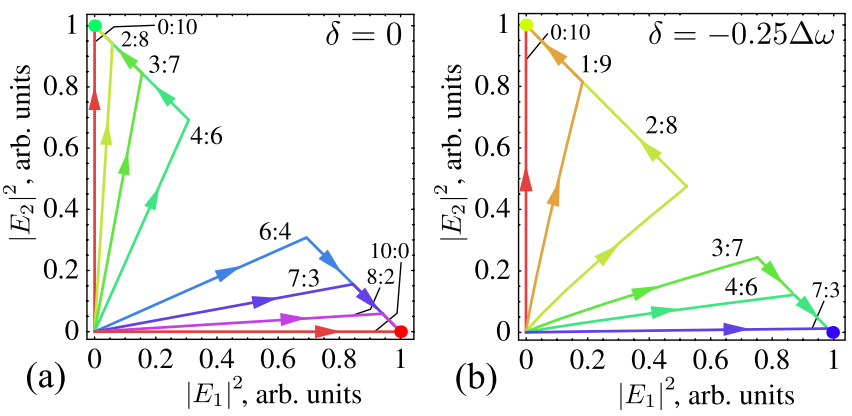

FIG. 2 (color online). Cavity phase diagrams for a lasing system governed by Eq. (1) for $F_{j}^{s} F(t) \gg F_{j}^{n}(t)$ for (a) symmetric $(\delta=0)$; and (b) nonsymmetric $(\delta=-0.25 \Delta \omega)$ values of the mode frequencies with respect to the central gain frequency $\omega_{a}\left[\omega_{a}<\omega_{0}\right.$, as shown in Fig. 1(b)]. The dots denote the stable cavity states, and the curves represent the phase trajectories for their temporal evolution for different ratios $F_{1}^{s}$ : $F_{2}^{s}$ in the direction of the arrows. 
case of symmetric detuning of the cavity modes with respect to the gain frequency ( $\delta=0)$, single-mode lasing is achieved into that mode whose spatial overlap with the seeding field $F_{j}^{s}$ is largest [Fig. 2(a)]. The asymmetry of the modes with respect to gain $(\delta \neq 0)$ shifts the turning point towards one of the modes, but if the seeding is chosen in a way that the spatial overlaps in Eq. (2) result in $F_{1}^{s} \gg F_{2}^{s}$ or $F_{1}^{s} \ll F_{2}^{s}$, each of the modes can nonetheless be selected for lasing [Fig. 2(b)].

To refine the predictions of this simple theory of bistable lasing we have modeled the lasing action in coupled $\mathrm{PhC}$ defects with a realistic injection mechanism (Fig. 1) using the finite-difference time-domain (FDTD) method [14,15]. The defect modes are located inside the $\mathrm{PhC}$ band gap. Both defects are filled with an active medium whose population dynamics are described at each space point by the rate equations of a four-level laser with an external pumping rate $W_{p}$. In defining the model and its parameter values we follow in detail Refs. $[15,16]$. In particular, the nonradiative transition times of this model are taken so as to achieve population inversion, i.e., $\tau_{32} \simeq \tau_{10} \ll \tau_{21}$, with $\tau_{32}=\tau_{10}=1 \times 10^{-13} \mathrm{~s}, \tau_{21}=3 \times 10^{-10} \mathrm{~s}$, and the total level population is $N_{\text {total }}=10^{24}$ per unit cell [16]. The Maxwell equations, supplemented by the usual equation of motion for the polarization density in the medium and by the laser rate equations [14-17], are solved for the geometry of Fig. 1(a) in TM polarization, where $\mathbf{E}(\mathbf{r}, t)=$ $E_{y}(x, z, t) \hat{\mathbf{y}}$. The seeding signal is excited by two emitters (linear groups of dipoles) engineered on the same chip as the $\mathrm{PhC}$ and is transmitted to the defects through waveguides in the PhC (see Fig. 1). Each of the emitters generates a single short Gaussian pulse with carrier frequency $\omega$ at or near $\omega_{a}$. The calculations have been performed using different, fixed values of the half-width duration $\sigma_{t}$ in the range between $\sigma_{t}=5 \times 10^{-14}$ and $5 \times 10^{-13}$. The relative phase of the fields in these pulses is chosen 0 or $\pi$. As expected, such seeding patterns almost exclusively excite the bonding and antibonding mode, respectively. Technically, the seeding dipoles are realized as pointlike oscillating current sources in the Maxwell equations [18]. Similarly, the spontaneous emission $[15,19,20]$ is modeled as an ensemble of point current sources, randomly placed in space, with temporally $\delta$-correlated Langevin noise [19]. For the FDTD computations the computational domain $13 a \times 13 a$ with perfectly matched layer boundary conditions was discretized with an $a / 16$ mesh and a time step of $d t=6 \times 10^{-17}$ s required by numerical stability; see [21] for details.

Neglecting noise at first, we find that, if the laser amplification line spectrally covers both resonant modes and provides a comparable effective gain for each of them (i.e., $|\delta|<\Delta \omega$ ), a seeding signal of the type described above can individually select any of the two modes when applied (with arbitrary strength) during the onset of lasing. The steady-state lasing is then nearly single mode, and the dominant mode is the one whose symmetry matches that of the seeding signal (Fig. 3). This is in agreement with the semianalytical theory described above. The electric field maps in Fig. 3 (bottom) show that the spatial field distributions of the two lasing modes remain nearly unaffected by the side-coupled seeding waveguides. In the present setup the laser output is primarily delivered through the seeding waveguides. A detailed study of the microlaser radiation characteristics will be given elsewhere. Moreover, we have shown that successive switchings from one mode to another are possible. To achieve this, the pump is first turned off to allow the currently lasing mode to decay. After a certain cooldown time $\tau_{c}$, the pumping is turned back on, and the cavity is reseeded for the other mode in the same way as the initial seeding occurred. Figure 3 demonstrates such a switching sequence from the bonding to the antibonding mode and back. The pump was kept on for a time needed to achieve quasicontinuous lasing, as confirmed by the temporal dependence of the cavity field. Looking at the spatial field distribution in this regime [panels (1)-(3) in Fig. 3], we make sure that the mode switching occurs in the desired order.

The switching time $\tau_{s}$ between two lasing modes is primarily controlled by the minimum cooldown time $\tau_{c \text {,min }}$, i.e., by the relatively slow mode decay time, $\tau=$ $1 / \kappa$, and hence by the cavity $Q$-factor $Q_{j}=\omega_{j} / \kappa$, not by the fast lasing onset after reseeding, $\tau_{s} \approx \tau_{c \text {,min }}$. Since the mode decays exponentially with time from its steady-state lasing amplitude $A_{0}$ and the reseeding signal must be strong enough to override the residue of the decaying initial mode, $\tau_{c, \text { min }}$ decreases logarithmically with increas-
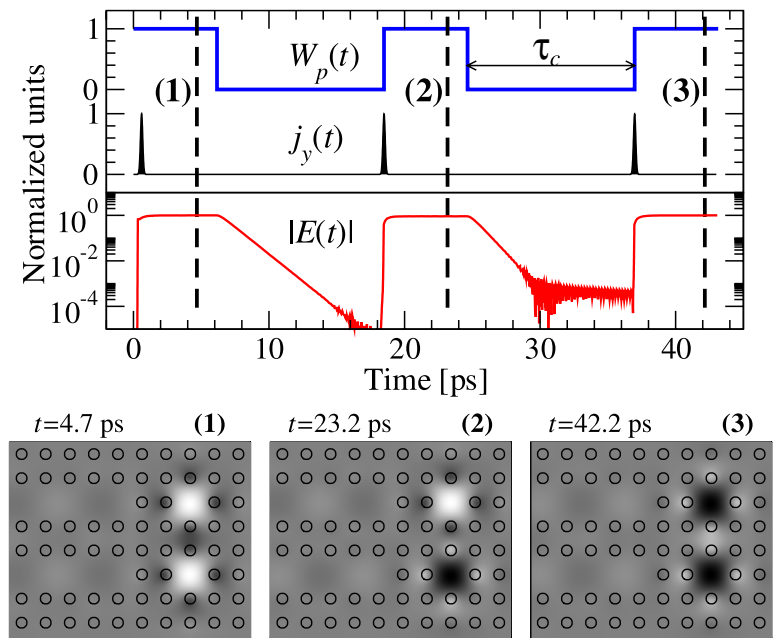

FIG. 3 (color online). Illustration of mode-to-mode switching. Top: time dependence of the normalized pumping power $W_{p}$, seeding signal $j_{y}$, and cavity field $E_{y}$. Bottom: spatial distribution of the electric field amplitude $E_{y}(x, z)$ (gray scale) in the steady-state lasing regime after initial seeding (1) and each reseeding (2),(3). The time instants (1)-(3) are marked in the top panel by dashed lines. This calculation was done for a typical pumping rate $W_{p}=1 \times 10^{13} \mathrm{~s}^{-1}$ and a seeding pulse duration $\sigma_{t}=1.2 \times 10^{-13} \mathrm{~s}$. 


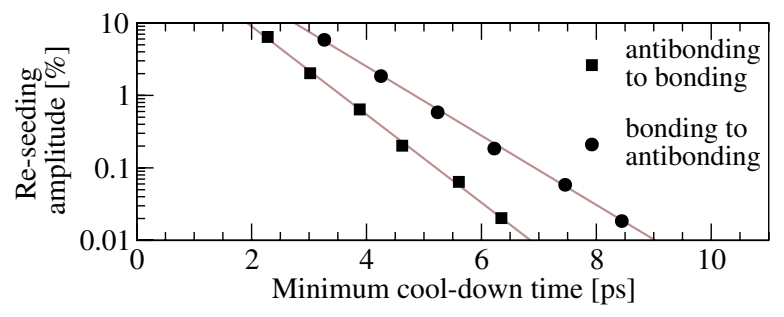

FIG. 4 (color online). The minimum switching time $\tau_{c \text {, min }}$ versus the reseeding amplitude, normalized to the saturated lasing mode amplitude. The seeding pulse duration is $\sigma_{t}=$ $0.12 \mathrm{ps}$. The cavity mode decay time is $\tau=0.7 \mathrm{ps}$. The longer $\tau_{c \text {, min }}$ for switching from the bonding to the antibonding mode is due to the nonzero gain detuning $\delta$ towards the bonding mode frequency [see Fig. 1(b)].

ing reseeding pulse power (i.e., with its time-integrated intensity) or, for a Gaussian pulse, with the seeding amplitude $S, \tau_{c \text {, min }}=-\left(c_{j} / \kappa\right) \ln \left(S / A_{0}\right)$. Here, $c_{j}$ is a dimensionless factor describing the coupling of the cavity mode $j=1,2$ to the laser transition. The corresponding numerical FDTD results are shown in Fig. 4. For realistic parameter values switching times of a few tens of picoseconds can be realized for reseeding amplitudes $S$ as low as $0.01 \%$ of the lasing mode amplitude $A_{0}, 2$ to 3 orders of magnitude faster than in previous devices [22].

When noise is present in the cavity, the FDTD calculations show that controlled switching is preserved as long as the integrated seeding power exceeds the noise power integrated over the lasing onset time. Otherwise, noise begins to dominate the lasing spectrum formation. In this case, the mode whose spatial overlap with the noise field at the onset of lasing is larger wins the competition. The same happens when the seeding pulse does not match the onset of lasing in time and its field residue has smaller amplitude in comparison to the noise field. These numerical observations are fully consistent with the analysis of the competition Eqs. (1) with the functions $F_{j}(t)$ given by Eq. (2). The switching is effective only if the influence of the seeding prevails in the resonator at the period of time when laser radiation starts to build up.

To summarize, the concept of switchable (rather than tunable) lasing in microstructures has been introduced. Instead of externally changing the parameters of a singlemode cavity, an inherently multimode cavity is used, and one of the modes is deliberately made to be dominant for lasing by means of injection seeding. This offers the possibility of all-optical frequency selection and switching in microlasers with particularly low switching times. As an example, we have investigated the mode switching in a system of two coupled defects in a 2D PhC lattice. For realistically chosen parameters a mode-to-mode switching on the picosecond scale has been numerically demonstrated. The results are consistent with a qualitative semianalytical model. It shows that a resonator supporting modes with similar spatial intensity profile tends towards bistability, which is the underlying physical mechanism of switchable lasing. The proposed concept is not limited to the model considered, but is expected to work in any resonator featuring bi- or multistability. Any coupled cavity based system would be a good candidate for the effects predicted. For instance, we have observed four-mode switching in coupled nanopillar waveguides of both periodic and nonperiodic longitudinal geometry [18].

This work was supported in part by DFG through SPP 1113 and FG 557 (S.V.Z., D. N. C., and J. K.) and by the EU Commission FP6 via project NewTon, NMP4-CT2005-017160 (A. V. L.).

*sergei@physik.uni-bonn.de

[1] K. J. Vahala, Nature (London) 424, 839 (2003).

[2] K. Yoshino et al., Appl. Phys. Lett. 75, 932 (1999); S. Mahnkopf et al., Appl. Phys. Lett. 82, 2942 (2003).

[3] W. Park and J.-B. Lee, Appl. Phys. Lett. 85, 4845 (2004); E. Schonbrun et al., IEEE Photonics Technol. Lett. 17, 1196 (2005).

[4] A. Figotin, Y. A. Godin, and I. Vitebsky, Phys. Rev. B 57, 2841 (1998); H. Yu et al., Opt. Express 13, 7243 (2005); E. P. Kosmidou, E. E. Kriezis, and T. D. Tsiboukis, IEEE J. Quantum Electron. 41, 657 (2005).

[5] A. Siegman, Lasers (University Science Books, Mill Valley, CA, 1986).

[6] W. Lee and W. R. Lempert, Appl. Opt. 42, 4320 (2003).

[7] S. E. Hodges et al., J. Opt. Soc. Am. B 14, 191 (1997).

[8] L. Florescu, K. Busch, and S. John, J. Opt. Soc. Am. B 19, 2215 (2002).

[9] N.B. Abraham, in Dynamics of Non-Linear Optical Systems, edited by L. Pesquera and F. J. Bermejo (World Scientific, Singapore, 1989), p. 3.

[10] M. M. Dignam et al., Phys. Rev. Lett. 96, 103902 (2006); D. P. Fussell et al., Phys. Rev. A 74, 043806 (2006).

[11] M. Tokushima, H. Yamada, and Y. Arakawa, Appl. Phys. Lett. 84, 4298 (2004); S. H. G. Teo et al., Photon. Nanostr. Fundam. Appl. 4, 103 (2006).

[12] G. P. Agrawal and N. K. Dutta, J. Appl. Phys. 56, 664 (1984); S. Ishii and T. Baba, Appl. Phys. Lett. 87, 181102 (2005).

[13] J. Bang, K. Blotekjar, and R. Ellingsen, IEEE J. Quantum Electron. 27, 2356 (1991).

[14] A. S. Nagra and R. A. York, IEEE Trans. Antennas Propag. 46, 334 (1998).

[15] X. Jiang and C. M. Soukoulis, Phys. Rev. Lett. 85, 70 (2000).

[16] S.-H. Chang and A. Taflove, Opt. Express 12, 3827 (2004).

[17] P. Bermel et al., Phys. Rev. A 74, 043818 (2006).

[18] S. V. Zhukovsky et al., Phys. Status Solidi B 244, 1211 (2007).

[19] G. M. Slavcheva, J. M. Arnold, and R.W. Ziolkowski, IEEE J. Quantum Electron. 10, 1052 (2004).

[20] M.-K. Seo et al., Opt. Express 13, 9645 (2005).

[21] A. V. Lavrinenko et al., Opt. Express 12, 234 (2004).

[22] O.A. Lavrova and D. J. Blumenthal, J. Lightwave Technol. 18, 1274 (2000). 